\title{
Optimal experiment design for identification of grey-box models
}

\author{
Sadegh, Payman; Melgaard, Henrik; Madsen, Henrik; Holst, Jan
}

\section{Published in:}

Proceedings of the American Control Conference

Link to article, DOI:

10.1109/ACC. 1994.751709

Publication date:

1994

Document Version

Publisher's PDF, also known as Version of record

Link back to DTU Orbit

Citation (APA):

Sadegh, P., Melgaard, H., Madsen, H., \& Holst, J. (1994). Optimal experiment design for identification of greybox models. In Proceedings of the American Control Conference (Vol. Volume 1, pp. 132-137). IEEE.

https://doi.org/10.1109/ACC.1994.751709

\section{General rights}

Copyright and moral rights for the publications made accessible in the public portal are retained by the authors and/or other copyright owners and it is a condition of accessing publications that users recognise and abide by the legal requirements associated with these rights.

- Users may download and print one copy of any publication from the public portal for the purpose of private study or research.

- You may not further distribute the material or use it for any profit-making activity or commercial gain

- You may freely distribute the URL identifying the publication in the public portal

If you believe that this document breaches copyright please contact us providing details, and we will remove access to the work immediately and investigate your claim 


\title{
Optimal Experiment Design for Identification of Grey-Box Models
}

\author{
P. SADEGH*, H. MELGAARD*, H. MADSEN* and J. HOLST ${ }^{\dagger}$ \\ * Inst. of Mathematical Modeling, Technical University of Denmark, \\ DK-2800 Lyngby, Denmark. \\ $\dagger$ Dept. of Electrical and Computer Engineering, University of Newcastle, \\ Newcastle, NSW 2308, Australia; On leave from: \\ Dept. of Mathematical Statistics, Lund Institute of Technology, \\ S-22100 LUND, Sweden.
}

\begin{abstract}
In this paper optimal experiment design is investigated for stochastic dynamic systems where the prior partial information about the system is given as a probability distribution function in the system parameters. The concept of information is related to entropy reduction in the system through Lindley's measure of average information, and the relationship between the choice of information related criteria and some estimators (MAP and MLE) is established. A continuous time physical model of the heat dynamics of a building is considered and the results show that performing an optimal experiment corresponding to a MAP estimation results in a considerable reduction of the experimental length. Besides, it is established that the physical knowledge of the system enables us to design experiments, with the goal of maximizing information about the physical parameters of interest.
\end{abstract}

\section{Introduction}

The term 'grey-box' is inspired by the use of 'blackbox' in connection with identification of systems where any knowledge of the system under consideration from other sources than measured data is not taken into account. In contrast, the grey-box identification is based upon the belief that partial knowledge about the actual system obtained from other sources in a beneficial manner can improve the result of the modeling procedure. Hence the partial information can influence the representation of the system, the design of experiments or the validation of the resulting model, just to mention a few of the points during the total modeling procedure where extra information might prove to be useful.

A basic reason for the interest in promoting the grey- box identification area is a desire to collect and systematize much of the procedures that earlier have been used to incorporate external knowledge, then often termed, e.g. physical modeling or mere intuition. The basic questions are then e.g. how the external knowledge is represented, how it is included in the modeling procedure as a whole or in a part of this procedure or what are the main advantages or disadvantages of the grey-box approach.

In recent years, some efforts have been reported to answer the questions around the grey-box modeling approach, see [1], [2], [3], and [4]. These references view different aspects of the same problem of incorporating partial prior knowledge in the identification and modeling procedure.

This paper focuses on the experiment design problem, i.e. on a part of the total grey-box modeling issue. This is a part where the partial knowledge may prove to be important, since it may help to design informative experiments with the goal of e.g. reducing the experiment time or decreasing the use of external excitation. However, this is also a very crucial issue, since the design is solely based on prior knowledge. The approach to the problem is statistically based. The concept of information is quantified using Lindley's measure of information, and the partial information is represented in a Bayesian framework as a prior distribution of the parameters that are to be estimated. This formulation of partial prior information is well suited to our adopted information criterion in the sense that the parameters are regarded as random variables. This also means that the prior information is embedded in a well founded procedure. Hence, it is possible within this procedure to discuss those qualities of the partial information that really are important, and to see also the limitations of the grey-box approach. It is also shown that this approach to experiment design leads to a more fundamental question about the whole experimental strategy, e.g. the 
choice of estimators. The same approach to the problem has been pursued in [5], [6], [7]. Finally, and equally important, this can be used to develop methods that for a given system can show where to try to obtain partial information in order to improve the total modeling and identification procedure.

The methods are applied to a physical model of heat dynamics of a building, where exploring the physical knowledge of the system is indispensable in order to estimate some physical parametric constants. It is then investigated how this collection of prior knowledge and partial prior information enables us to design efficient informative experiments.

\section{Experiment Design For Grey-Box Models}

The basic concept behind the design of optimal experiments is to maximize a function which is a measure of the "goodness" of those experiments.

In a traditional approach, one may regard a suitable scalar function of Fisher's information matrix as the criterion. This is related to maximization of the posterior precision of the unknown parameters, where the posterior precision is merely obtained by performing measurements during the experiment and processing the data statistically. However, the purely statistical processing of data is in many cases not compatible with the physical reality [4].

In this paper, MAP estimator is introduced as a grey-box estimator. However, the grey-box approach can also be used in connection with other estimators where partial prior information about the value of the estimates does not exist or is ignored in the estimation procedure, e.g. in connection with (unconstrained) ML estimator. The latter approach has been thoroughly discussed in [8].

It is well known that if $Y$ is a set of i.i.d. random variables the covariance of any unbiased efficient estimator of the unknown parameter $\beta$ will asymptotically reach the lower bound of the Cramer-Rao inequality given by the inverse of Fisher's information matrix [9]. However, in finite experiment time, the posterior precision will be dependent upon the choice of the efficient estimator.

Fisher's information matrix is defined as

$$
M_{F}=E_{Y \mid \beta}\left\{\left(\frac{\partial \log p(Y \mid \beta)}{\partial \beta}\right)^{T}\left(\frac{\partial \log p(Y \mid \beta)}{\partial \beta}\right)\right\}
$$

where $p(Y \mid \beta)$ is the probability density function of the random variable $Y$, given $\beta$. The following theorem establishes a relation between the posterior covariance of the parameters and Fisher's information matrix when a MAP estimator is used.

Theorem 2.1 Assume that the prior information about system parameters is embodied in a Gaussian distribution with covariance $\Sigma_{p r e}$. Also assume that a MAP estimator is used to estimate the unknown parameters based on sampled observations for a model brought into the regression form.

$$
y_{t}=\varphi_{t}^{T} \beta+\epsilon_{t}
$$

where $\left\{\epsilon_{t}\right\}$ is a sequence of Gaussian distributed random variables with known covariance, uncorrelated with $\left\{\varphi_{t}\right\}$.

Then the posterior covariance matrix $\Sigma_{\text {post }}$ is given by

$$
\Sigma_{p o s t}^{-1}=\Sigma_{p r e}^{-1}+M_{F}
$$

or equivalently

$$
\Sigma_{p o s t}^{-1}=\Sigma_{p r e}^{-1}+N \bar{M}_{F}
$$

where $M_{F}$ is Fisher's information matrix, $\bar{M}_{F}$ the average information matrix, and $N$ the length of experiment.

Proof: See [7]

The theorem is stated under very restrictive assumptions, e.g. that the output should be written as a linear regression in parameters. However, it can be shown that the theorem is true in more general cases. For a formal proof see [6].

The MAP estimator is asymptotically efficient, since the posterior covariance matrix approaches the lower bound of the Cramer-Rao inequality for $N \rightarrow \infty$, see (3).

If the posterior information is obtained applying a ML estimator, $\Sigma_{p r e}$ should be set equal to $\infty$ since ML and MAP estimators coincide in case the prior distribution of parameters is non-informative [10].

\section{Information Theoretic Approach}

In this section, the concept of information is related to Lindley's measure of information. The information related criterion is shown to depend upon the choice of estimator.

Definition 3.1 The entropy of a random variable $X$ having probability density function $p(X)$ is defined to be

$$
H_{x}=-E_{X}[\log p(X)]
$$


Definition 3.2 Lindley's measure of the average amount of information provided by an experiment $\varepsilon$ with data $y$ and parameters $\beta$ is defined to be

$$
\mathcal{J}(\varepsilon)=H_{\beta}-E_{y}\left[H_{\beta \mid y}\right]
$$

Theorem 3.1 With the same assumptions as in Theorem 2.1, maximizing Lindley's measure of the average amount of information, $\mathcal{J}(\varepsilon)$, is equivalent to solving the optimization problem

$$
\begin{aligned}
& \min _{\varepsilon} J \\
& J=-E_{\beta}\left[\log \operatorname{det}\left\{\Sigma_{p r e}^{-1}+M_{F}\right\}\right]
\end{aligned}
$$

Proof: We regard the estimation as a means by which further information about the system parameters is provided. Recalling that MAP is the mode of the posterior distribution, the maximum amount of information with respect to Lindley's measure is obtained by $M A P$ for each experiment.

Since the prior distribution of the parameters and the distribution of observations given $\beta$ is Gaussian and the covariance of the residuals is known, the posterior distribution of the parameters will also be Gaussian. Let's denote the posterior mean and covariance by $\bar{\beta}$ and $\Sigma_{\text {post }}$. From (2) we have

$$
\Sigma_{p o s t}^{-1}=\Sigma_{p r e}^{-1}+M_{F}
$$

From Definition 3.2

$$
\begin{aligned}
& \mathcal{J}(\varepsilon) \\
& =-E_{\beta}\{\log p(\beta)\}+E_{y} E_{\beta \mid y}\{\log p(\beta \mid y)\} \\
& =-E_{\beta}\{\log p(\beta)\}+E_{y} E_{\beta \mid y}\left\{-\frac{n_{p}}{2} \log (2 \pi)\right\} \\
& -E_{y} E_{\beta \mid y}\left\{\frac{1}{2} \log \operatorname{det} \Sigma_{\text {post }}\right. \\
& \left.+\frac{1}{2}(\beta-\bar{\beta})^{T} \Sigma_{\text {post }}^{-1}(\beta-\bar{\beta})\right\}
\end{aligned}
$$

As all the other terms obviously are constant, we only focus on the last two terms

$$
\begin{aligned}
& E_{y} E_{\beta \mid y}\left\{\frac{1}{2} \log \operatorname{det} \Sigma_{\text {post }}\right\} \\
& =E_{\beta} E_{y \mid \beta}\left\{\frac{1}{2} \log \operatorname{det} \Sigma_{\text {post }}\right\} \\
& =E_{\beta}\left\{\frac{1}{2} \log \operatorname{det} \Sigma_{\text {post }}\right\} \\
& =-E_{\beta}\left\{\frac{1}{2} \log \operatorname{det} \Sigma_{\text {post }}^{-1}\right\}
\end{aligned}
$$

The other term can be written as

$$
\begin{aligned}
& E_{y} E_{\beta \mid y}\left\{\frac{1}{2}(\beta-\bar{\beta})^{T} \Sigma_{p o s t}^{-1}(\beta-\bar{\beta})\right\} \\
& =E_{y} E_{\beta \mid y}\left\{\frac{1}{2} \operatorname{trace}^{-1} \Sigma_{\text {post }}^{-1}(\beta-\bar{\beta})(\beta-\bar{\beta})^{T}\right\} \\
& =E_{y}\left\{\frac{1}{2} \operatorname{trace}_{\text {post }}^{-1} E_{\beta \mid y}\left[(\beta-\bar{\beta})(\beta-\bar{\beta})^{T}\right]\right\} \\
& =E_{y}\left\{\frac{n_{p}}{2}\right\} \\
& =\frac{n_{p}}{2}
\end{aligned}
$$

where $n_{p}$ is the number of parameters. Now using (2) establishes the theorem.
In many practical applications, an approximation of the mean value in (4) is computed by setting the parameters equal to their prior mean values. This approximation simplifies the study considerably. Thus depending on the estimation method or the choice of a Bayesian or non-Bayesian criterion, the following criteria would be of interest:

$$
\begin{aligned}
& J_{1}=-\left[\log \operatorname{det}\left(\bar{M}_{F}\right)\right]_{\beta=E\{\beta\}} \\
& J_{2}=-\left[\log \operatorname{det}\left(N \bar{M}_{F}+\Sigma_{p r e}^{-1}\right)\right]_{\beta=E\{\beta\}} \\
& J_{3}=-E_{\beta}\left[\log \operatorname{det}\left(\bar{M}_{F}\right)\right] \\
& J_{4}=-E_{\beta}\left[\log \operatorname{det}\left(N \bar{M}_{F}+\Sigma_{\text {pre }}^{-1}\right)\right]
\end{aligned}
$$

These criteria demonstrate different levels of including partial prior information about parameter values. Optimization with respect to $J_{1}$ results in designs which are strongly dependent upon the prior information. The dependence is even more pronounced in $J_{2}$. Therefore, it might be beneficial to perform a sensitivity analysis, i.e. to determine the sensitivity of the design to changes in parameters, when using these criteria. An alternative approach could be choosing Bayesian design criteria like $J_{3}$ and $J_{4}$.

Table 1 summarizes the relation between the choice of estimators and the optimization criterion, expressed in both a Bayesian and non-Bayesian form.

Table 1: Summary of the optimality criteria.

\begin{tabular}{||c|c|c||}
\hline & ML & MAP \\
\hline Non-Bayesian & $J_{1}$ & $J_{2}$ \\
\hline Bayesian & $J_{3}$ & $J_{4}$ \\
\hline
\end{tabular}

\section{Frequency Domain Design}

Consider a linear discrete time system of the form

$$
y_{t}=G_{1}(q) u_{t}+G_{2}(q) \epsilon_{t}
$$

where $\left\{u_{t}\right\}$ and $\left\{y_{t}\right\}$ are the input and output sequences, respectively, and $\left\{\epsilon_{t}\right\}$ is a sequence of i.i.d. Gaussian random variables having zero mean value and covariance $\Sigma$. $G_{1}(q)$ and $G_{2}(q)$ are transfer functions where $q$ is the forward shift operator. For simplicity, restrict attention to a single input, single output case with $G_{2}(\infty)=1$.

Assume that

1) The experiment time is large.

2) The input $\left\{u_{t}\right\}$ is restricted to the class of signals, admitting a spectral representation with spectral distribution function $F(\omega), \omega \in[-\pi, \pi]$.

3) The constraint is taken to be the allowable input 
power.

Defining $\xi$ as

$$
d \xi(\omega)= \begin{cases}\frac{1}{2} d F(\omega) & \omega=0 \cup \omega=\pi \\ d F(\omega) & \omega \in] 0, \pi[\end{cases}
$$

the power restriction of the input signal can be stated by

$$
P_{u}=\frac{1}{\pi} \int_{0}^{\pi} d \xi(\omega)=1
$$

It can be shown that the set of all average information matrices is the convex hull of the average information matrices corresponding to single frequency designs [10]. Based on this fact, the following theorem can be proved.

Theorem 4.1 For the design criteria $J_{1}, J_{2}, J_{3}$, and $J_{4}$ given in (5), optimal designs exist comprising not more than $\frac{n_{p}\left(n_{p}+1\right)}{2}$ sinusoidal components, where $n_{p}$ is the number of parameters.

PRoOF: See [10] for a proof in the non-Bayesian case and $[5,8]$ for a proof in the Bayesian case.

Knowing that a finite number of sinusoidal components suffice to reach optimality, numerical optimization algorithms can be easily applied to find the frequencies corresponding to the optimal input sinusoidal components and their corresponding power shares.

For instance corresponding to the criterion $J_{4}$, one can formulate the optimization problem

$$
\begin{array}{rl}
\min _{\alpha_{1}, \ldots, \alpha_{n}, \omega_{1}, \ldots, \omega_{n}} & f\left(\alpha_{1}, \ldots, \alpha_{n}, \omega_{1}, \ldots, \omega_{n}\right) \\
\sum_{i=1}^{n} \alpha_{i}=1 & \\
0 \leq \alpha_{i} \leq 1 \quad i=1, \ldots, n \\
\omega_{l} \leq \omega_{i} \leq \omega_{u} \quad i=1, \ldots, n
\end{array}
$$

where the cost function is

$$
\begin{array}{r}
f\left(\alpha_{1}, \ldots, \alpha_{n}, \omega_{1}, \ldots, \omega_{n}\right)= \\
-E_{\beta} \log \operatorname{det}\left[\Sigma_{p r e}^{-1}+\sum_{i=1}^{n} N \bar{M}_{F}\left(\omega_{i}\right) \alpha_{i}\right]
\end{array}
$$

$\bar{M}_{F}\left(\omega_{i}\right)$ is the average information matrix corresponding to single input frequency $\omega_{i}$, and $\omega_{l}$ and $\omega_{u}$ are our initial guess of lower and upper bounds of the optimal design frequencies. $n$ is the maximum number of sinusoidal components which suffice for optimality. In many cases $n$ is less than the number $\frac{n_{p}\left(n_{p}+1\right)}{2}$ which is predicted by Theorem (4.1). The methods of including the prior knowledge by considering Bayesian criteria are thoroughly investigated in
[6] and [5].

The technique can be extended to continuous time models with minor modifications: replace the forward shift operator with differentiation operator, and the frequency range $[0, \pi]$ with $[0,+\infty]$.

In the following, the application of MAP estimation and its corresponding optimal experiment based on a physical model of the heat dynamics of a building are illustrated.

\section{Application}

This application shows how the optimal design depends upon the choice of the estimators and the prior information about the system parameters. It also illustrates that the physical knowledge of the system enables us to design experiments which are informative about the physical parameters of interest.

Throughout the example, the non-Bayesian criteria are considered. The Bayesian versions of the criteria can be investigated as well. However, previous experiments on the model show that the optimal input spectrum exhibits small sensitivity to perturbations in parameter values. Hence, a Bayesian study will in this case only contribute to computational complexity. Besides, there are some other interesting aspects of experimental design that can be studied, even within a non-Bayesian framework, e.g. a study of experimental design using MAP estimators, and these aspects are outlined in the application as follows.

The following set of stochastic differential equations, which originates from physical considerations, provides a model of heat dynamics of a building [11].

$$
\begin{aligned}
& {\left[\begin{array}{c}
d T_{m} \\
d T_{i}
\end{array}\right]=\left[\begin{array}{cc}
\frac{-1}{R_{i} C_{m}} & \frac{1}{R_{i} C_{m}} \\
\frac{1}{R_{i} C_{i}} & -\left(\frac{1}{R_{a} C_{i}}+\frac{1}{R_{i} C_{i}}\right)
\end{array}\right]\left[\begin{array}{c}
T_{m} \\
T_{i}
\end{array}\right] d t} \\
& +\left[\begin{array}{ccc}
0 & 0 & \frac{A_{w} p_{s}}{C_{m}} \\
\frac{1}{R_{a} C_{i}} & \frac{1}{C_{i}} & \frac{A_{w}\left(1-p_{s}\right)}{C_{i}}
\end{array}\right]\left[\begin{array}{c}
T_{a} \\
Q_{h} \\
Q_{s}
\end{array}\right] d t \\
& +\left[\begin{array}{c}
d w_{m}(t) \\
d w_{i}(t)
\end{array}\right]
\end{aligned}
$$

with the following parameters

- $R_{i}$ The resistance between the room air and the large heat accumulating medium.

- $R_{a}$ The resistance between inner walls and the ambient air.

- $C_{i}$ The capacity of the small heat accumulating medium (inner part of the walls).

$-C_{m}$ The capacity of the large heat accumulating medium (due to concrete flags placed on the floor).

The indoor temperature is measured, and both process and measurement noise terms are considered. - $T_{i}$ The temperature of the small heat accumulating medium (indoor temperature). 
- $T_{m}$ The temperature of the large heat accumulating medium.

- $A_{w}$ Effective window area.

- $p_{s}$ The part of solar radiation which reaches the building.

- $T_{a}$ The ambient air temperature.

- $Q_{h}$ Heat supplied by heater (controlled input).

- $Q$ s Heat supplied by solar radiation.

- $w_{m}(t), w_{i}(t)$ Elements of a vector Wiener process.

Assume that the vector

$$
\beta=\left[\begin{array}{c}
R_{i} \\
R_{a} \\
C_{i} \\
C_{m}
\end{array}\right]
$$

is chosen as the parameter vector, i.e. the goal of the experiment is to gain information about the elements of $\beta$. In other words, the problem of designing the optimal experiments can be formulated as follows. Find an input signal, expressed as a function of time or specified by its power spectrum, that maximizes information about $\beta$, or a sub-set of $\beta$, according to some suitable information measure, when the means by which the behavior of the system can be observed is recording the indoor temperature (corrupted with noise) as the system's response to excitations in the indoor heat flow supplied by the heater.

In $[10]$, methods to derive the information matrix are presented, and in [5] these methods are extended and applied to the model of heat transfer in a building, where the model is non-linear in parameters. Experiments are suggested which minimize the posterior uncertainty in the elements of $\beta$.

For some typical values of the thermal parameters:

$$
\begin{aligned}
& m_{R_{a}}=0.4788\left({ }^{\circ} \mathrm{C} / K W\right) \\
& m_{R_{a}}=29.38\left({ }^{\circ} \mathrm{C} / K W\right) \\
& m_{C_{1}}=1.183\left(K W h /{ }^{\circ} \mathrm{C}\right) \\
& m_{C_{m}}=3.987\left(K W h /{ }^{\circ} \mathrm{C}\right)
\end{aligned}
$$

and some typical values of variances of process and measurement noise terms, an optimal power distribution shown in Fig. 1 is obtained. The design is based on the criterion $J_{1}$. The figure shows that the optimal power distribution has components both in low and high frequencies, and it corresponds to a slow and a fast dynamics in the system. The slow dynamics is due to the large capacitor $C_{m}$, and the fast dynamics is due to the small capacitor $C_{i}$.

Now the role of adding prior information is studied by considering different prior uncertainties and using the design criterion related to the MAP estimator. By large uncertainty in $C_{i}$, we mean that the prior variances of the parameters are given by

$$
\begin{array}{ll}
\sigma_{R_{i}}^{2}=10^{-4} & \sigma_{R_{a}}^{2}=10^{-4} \\
\sigma_{C_{2}}^{2}=10^{-1} & \sigma_{C_{m}}^{2}=10^{-6}
\end{array}
$$

and by large uncertainty in $C_{m}$, they are given by

$$
\begin{array}{ll}
\sigma_{R_{i}}^{2}=10^{-4} & \sigma_{R_{a}}^{2}=10^{-4} \\
\sigma_{C_{i}}^{2}=10^{-6} & \sigma_{C_{m}}^{2}=10^{-1}
\end{array}
$$

Considering $J_{2}$ and the prior variances given by (13), the optimal power distribution is shown in Fig. 2.

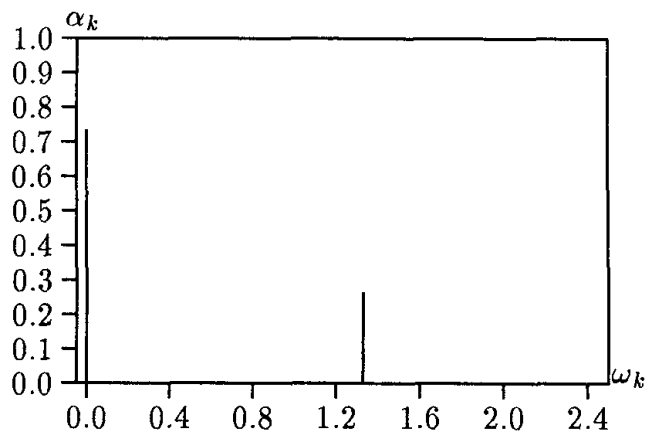

Figure 1: Optimal power distribution for building modeling, criterion $J_{1}$.

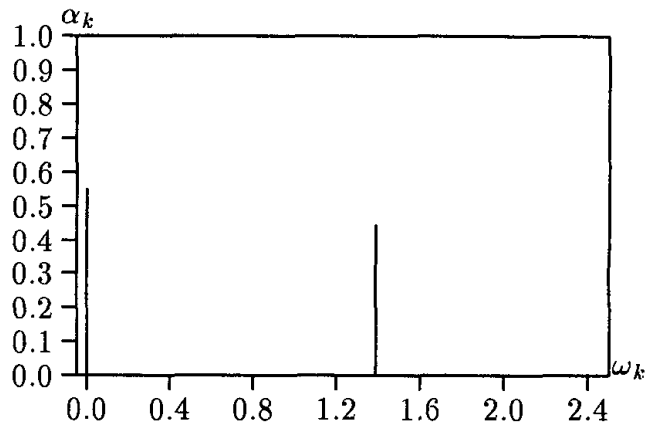

Figure 2: Optimal power distribution for building modeling, criterion $J_{2}$, large uncertainty on $C_{i}$.

It is interesting to note that the optimal input is informative about the most uncertain capacity, since it shifts power to higher frequencies. When the uncertain parameter is the larger capacity, optimizing $J_{2}$ will lead to a design' which solely consists of a DCcomponent, i.e. a drastic shift of power towards lower frequencies. In the assessment that follows later, it is illustrated how the designs obtained by optimizing $J_{2}$, where an extra amount of prior information is added, together with using the MAP estimator result in a reduction of the experiment time relative to the design obtained by minimizing $J_{1}$ and using the ML estimator. However, notice that misguiding results could be caused by wrongly or ill-specified prior information. For instance, if the smaller capacity is quite different from our prior guess, very little information about its value is provided by a DC-excitation, and the posterior estimate of the capacity will shift only slightly from the initial wrong value. This flaw can not be 
assigned only to the experimental design, but more generally to a wrong choice of the whole experimental strategy, which among others covers the choice of an unsuitable estimator and its corresponding experiment.

Denoting the optimal design by $\xi^{*}$, we define $P A V$ by

$$
\left.P A V=\left.\left(\operatorname{det}\left(\Sigma_{p o s t}\left(\beta, \xi^{*}\right)\right)\right)\right|_{\beta=E\{\beta\}}\right)^{\left(\frac{1}{n_{p}}\right)}
$$

as a measure by which it is possible to compare the posterior precisions of different sets of estimates for a specific experiment time. This is equivalent to comparing the mean volumes of the posterior confidence ellipsoids for the parameters, where the posterior knowledge has been gained under different conditions. Note that, prior to the experiment, this mean volume is approximated by setting the parameters equal to their prior mean values. The experiments can be compared either by comparing the experiment lengths for a predefined $P A V$ or by comparing the $P A V$ s for a predefined experiment time. A study of the results in Table 2 and Table 3 show that an optimal experiment corresponding to the criterion $J_{2}$ together with the MAP estimation shorten $N$, the assessed length of experiment, considerably.

Table 2: Experimental design results for a building, ML estimator. $P A V \mathrm{~s}$ are scaled by $10^{6}$.

\begin{tabular}{||c|c|c||}
\hline \multicolumn{3}{|c||}{$M L E$} \\
\hline Prior variances & $N=1540 h$ & $P A V=2.8$ \\
\hline$(13)$ & $P A V=18$ & $N=10000 h$ \\
\hline$(14)$ & $P A V=18$ & $N=10000 h$ \\
\hline
\end{tabular}

Table 3: Experimental design results for a building, MAP estimator. $P A V$ s are scaled by $10^{6}$.

MAP estimator. $P A V$ s are scaled by $10^{6}$.
\begin{tabular}{||c|c|l||}
\hline \multicolumn{3}{|c||}{$M A P$} \\
\hline Prior variances & $N=1540 h$ & $P A V=2.8$ \\
\hline$(13)$ & $P A V=2.8$ & $N=1540 h$ \\
\hline$(14)$ & $P A V=3.6$ & Not computed \\
\hline
\end{tabular}

\section{CONCLUSION}

In the paper, different levels of including prior information about the system, for experiment design, have been discussed. The different levels, which are summarized in Table 1, include the use of the MAP and ML estimators as well as Bayesian and non-Bayesian criteria for optimality of the experiment. Besides, it is shown that the physical knowledge of the system enables us to design experiments which are informative about the physical parameters of interest. The application of experimental design for grey-box models to the heat dynamics of a building illustrates the role of the physical knowledge and shows how the choice of estimators affects the optimal design. By considering the MAP estimator the optimal design is dependent upon the prior uncertainty of the parameters, and in general the total experiment time is significantly shortened. The application demonstrates the importance of using grey-box methods for experiment design.

\section{References}

[1] T. Bohlin. Computer-aided grey-box validation. Technical Report TRITA-REG-8403, Dept of Automatic Control, Royal Institute of Technology, Stockholm, Sweden, 1984.

[2] S. Graebe. Theory and Implementation of Gray Box Identification. PhD thesis, Dept of Automatic Control, Royal Institute of Technology, Stockholm, Sweden, 1990.

[3] J. Holst, U. Holst, H. Madsen, and H. Melgaard. Validation of grey box models. In IFAC Symposium on Adaptive Control and Signal Processing 92, pages $407-414,1992$.

[4] Herbert J. A. F. Tulleken. Grey-box modelling and identification using physical knowledge and bayesian techniques. Automatica, 29(2):285-308, 1993.

[5] Payman Sadegh. Experiment design for grey box models. Master's thesis, Institute of Mathematical Statistics and Operations Research, Technical University of Denmark, Lyngby, Denmark, 1993.

[6] P. Sadegh, J. Holst, H. Madsen, and H. Melgaard. Experiment design and estimation for greybox models. Technical Report EE9401, Department of Electrical Engineering and Computer Science, The University of Newcastle, Newcastle, Australia, 1994.

[7] P. Sadegh, H. Melgaard, H. Madsen, and J. Holst. On the usefulness of grey-box information when doing experiment design for system modeling and identification. In 10th IFAC Symposium on System Identification and Parameter Estimation, 1994.

[8] H. Melgaard, P. Sadegh, H. Madsen, and J. Holst. Experiment design for grey-box models. In Preprints of the 12th IFAC World Congress, Sydney, volume 2, pages 145-148, 1993.

[9] C. R. Rao. Linear Statistical Inference and Its Applications. 2nd edition, Wiley, 1973.

[10] Graham C. Goodwin and Robert L. Payne. Dynamic System Identification: Experiment Design and Data Analysis. Academic Press, 1977.

[11] Henrik Madsen, Henrik Melgaard, and Jan Holst. Identification of building performance parameters. In J.J. Bloem, editor, Workshop on Advanced Identification Tools in Solar Energy Research, Non Nuclear Energy, pages 37-60. Commission of the European Communities, DG XII, 1990. 